\section{Case Report}

Check for updates

\section{OPEN ACCESS}

Received: Aug 26, 2019

Revised: Nov 2, 2019

Accepted: Nov 22, 2019

Jurado CA, Tsujimoto A, Watanabe $\mathrm{H}$, Villalobos-Tinoco J, Garaicoa JL, Markham MD, Barkmeier WW, Latta MA.

*Correspondence to

Akimasa Tsujimoto, DDS, PhD

Assistant Professor, Department of Operative Dentistry, Nihon University School of Dentistry, 1-8-13, Kanda-Surugadai, Chiyoda-ku, Tokyo 101-8310, Japan.

E-mail: tsujimoto.akimasa@nihon-u.ac.jp

Copyright $($ 2020. The Korean Academy of Conservative Dentistry

This is an Open Access article distributed under the terms of the Creative Commons Attribution Non-Commercial License (https:// creativecommons.org/licenses/by-nc/4.0/) which permits unrestricted non-commercial use, distribution, and reproduction in any medium, provided the original work is properly cited.

Conflict of Interest

No potential conflict of interest relevant to this article was reported.

\title{
Chair-side CAD/CAM fabrication of a single-retainer resin bonded fixed dental prosthesis: a case report
}

\author{
Carlos Alberto Jurado $\left(\mathbb{D},{ }^{1}\right.$ Akimasa Tsujimoto $\mathbb{1}, 2^{2^{*}}$ Hidehiko Watanabe $\mathbb{1},,^{3}$ \\ Jose Villalobos-Tinoco (1), Jorge Luis Garaicoa $(1),{ }^{3}$ Mark David Markham (1), ${ }^{5}$ \\ Wayne Walter Barkmeier (10,5 Mark Andrew Latta $\left(\mathbb{C}^{5}\right.$
}

'Clinical Digital Dentistry, A.T. Still University Arizona School of Dentistry \& Oral Health, Mesa, AZ, USA ${ }^{2}$ Department of Operative Dentistry, Nihon University School of Dentistry, Tokyo, Japan ${ }^{3}$ Department of Restorative Dentistry, Oregon Health and Science University School of Dentistry, Portland, OR, USA

${ }^{4}$ Department of Oral Rehabilitation, Autonomous University of Queretaro School of Dentistry, Queretaro, Mexico ${ }^{5}$ Department of General Dentistry, Creighton University School of Dentistry, Omaha, NE, USA

\section{ABSTRACT}

This clinical report describes designing and fabricating a single-retainer resin-bonded fixed dental prosthesis with a chair-side computer-aided design/computer-aided manufacturing system. The whole procedure, from tooth extraction to final placement of the prosthesis, was completed in one day, and a single clinic visit. No clinical complications were found at the 2-year follow-up after placement of the restoration, and satisfactory functional and esthetic results were achieved.

Keywords: Atraumatic restorative treatment; Dental prosthesis; Etching

\section{INTRODUCTION}

Replacing a single missing tooth among the anterior teeth is challenging for the clinician, and many restorative options are currently available [1]. Although implant therapy is becoming the ideal option for patients who have an appropriate amount of alveolar bone [2], adhesive bridges offer less invasive clinical options than either implant therapy or conventional crown-retained prosthetics [3]. The history of adhesive bridges is believed to have started with the restorations developed by Rochette in 1973 [4]. Rochette designed a wing-shape gold alloy perforated abutment device to support and fix a prosthetic anterior mandibular tooth and bonded prosthesis to the lingual side of the abutment teeth using methyl methacrylate (MMA) resin. This allowed for a more conservative preparation of the abutment teeth than that required for cemented conventional crown-retained prosthetics. The clinical methodology evolved over the following years. In 1977, Howe and Denehy used nickel-chrome alloys for a fixed prosthesis, which are stronger than gold alloys, and replaced MMA with packable resin composites, for increased durability [5]. In the 1980s, Livaditis extended the technique to molars and used electrical etching rather than perforations to create the bonding surface [6,7]. This was combined with the use of flowable resin composites for the bonding procedure. From the early 1990s, high strength ceramic materials, including lithium disilicate and zirconia, began 


\section{Author Contributions}

Conceptualization: Jurado CA, Tsujimoto A. Data curation: Jurado CA, Villalobos-Tinoco J, Garaicoa JL. Project administration: Watanabe H, Latta MA. Software: Jurado CA. Supervision: Tsujimoto A. Writing - original draft: Jurado CA. Writing - review \& editing: Tsujimoto A, Markham MD, Barkmeier WW.

ORCID IDs

Carlos Alberto Jurado (D)

https://orcid.org/0000-0001-7437-4855

Akimasa Tsujimoto (D)

https://orcid.org/0000-0002-7304-3971

Hidehiko Watanabe (iD)

https://orcid.org/0000-0002-0390-2312

Jose Villalobos-Tinoco (iD

https://orcid.org/0000-0003-2156-8335

Jorge Luis Garaicoa (D)

https://orcid.org/0000-0003-4050-7597

Mark David Markham (iD)

https://orcid.org/0000-0002-4167-4541

Wayne Walter Barkmeier (D)

https://orcid.org/0000-0001-7256-3150

Mark Andrew Latta (D)

https://orcid.org/0000-0002-2859-2680 to replace metals as the material for the framework, and resin-bonded fixed dental prostheses (RBFDPs) approached their current form [8].

There are two options for the structure of RBDFPs: 1) single-retainer RBDFPs; and 2) 2-retainer RBDFPs. A recent systematic review of the literature of clinical studies of all ceramic RBFDPs showed that the estimated 5-year survival rate was $91.2 \%$, and that singleretainer RBFDPs had a higher survival rate $(p<0.01)$, lower debonding rate, $(p<0.05)$, and lower fracture rate $(p<0.01)$ than two-retainer equivalents. Thus, single-retainer RBFDPs are currently the preferred treatment [9]. The most important advantages of single-retainer RBFDPs are the simplicity of the minimally invasive preparation design, reduced financial costs, and better clinical durability [10]. The application of RBFDPs has increased in recent years, but there are no clinical reports of the digital design and fabrication of such prostheses using chair-side computer-aided design/computer-aided manufacturing (CAD/CAM) systems.

The following clinical report describes the steps involved in designing and milling a singleretainer RBFDP for an edentulous site with a common chair-side CAD/CAM system in a single clinic visit.

\section{CASE REPORT}

A 55-year-old female patient presented to the clinic with a chief complaint of a fractured tooth and wanted treatment to restore the site. After the initial evaluation, it was diagnosed that tooth \#6 was fractured at the level of the gingival tissue and was not restorable (Figure 1). The patient was informed of the treatment options available of a removable partial denture, a fixed prosthesis, or implant therapy, and the patient chose to have an implant after tooth extraction. The patient intended to have an aesthetic and durable temporary prothesis placed not only during the period when the extraction site was healing, but also cost savings for implant treatment. The patient was informed of the possibility of fabricating a single retainer cantilevered RBFDP as a long-term provisional restoration using a chair-side CAD/CAM system (CEREC, Dentsply Sirona, York, PA, USA), and accepted his treatment modality.

First, impressions were made with polyvinyl siloxane material (Virtual, Ivoclar Vivadent, Schaan, Liechtenstein), and master cast models were fabricated with type IV stone (Fujirock, GC, Tokyo, Japan). Using the stone model, the minimal coronal portion of the fractured tooth was removed with a diamond bur and a slight concavity was creating. The master cast models were scanned, and the software indications for this type of restoration was as a fixed partial
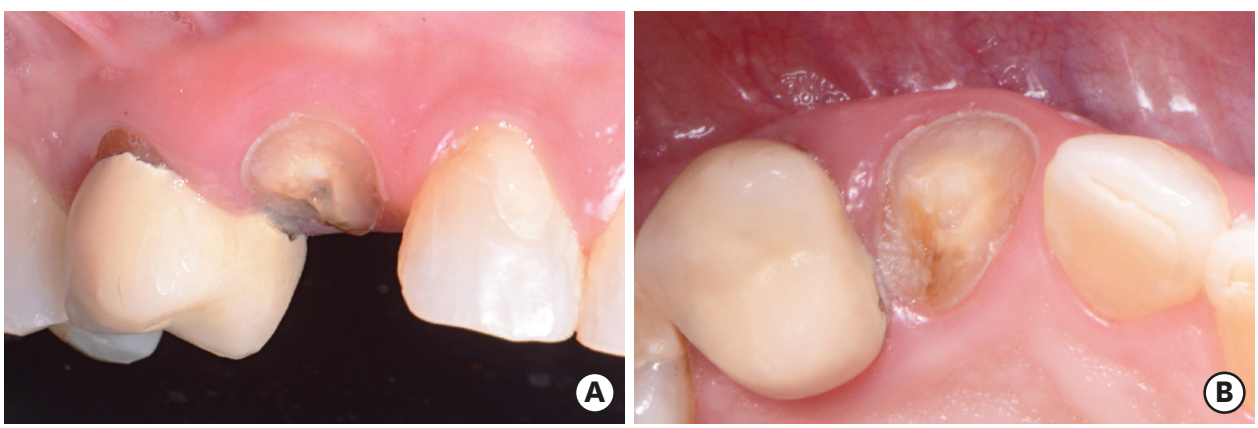

Figure 1. Patient presented with a non-restorable fractured tooth \#6. (A) Preoperative facial view. (B) Preoperative occlusal view. 

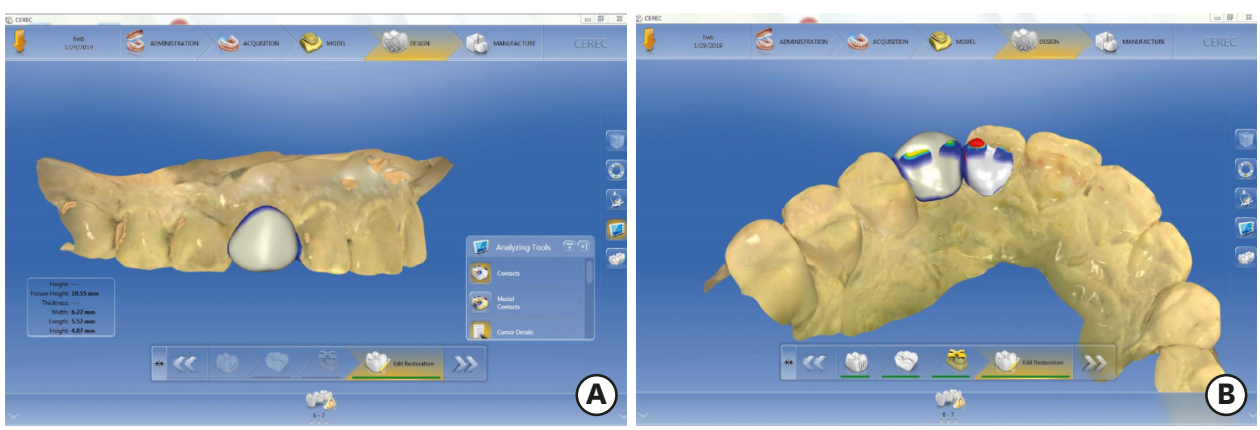

Figure 2. Scanning the diagnostic casts and designing of the restoration. (A) Facial view of digitally designed restoration. (B) Occlusal view of digitally designed restoration.

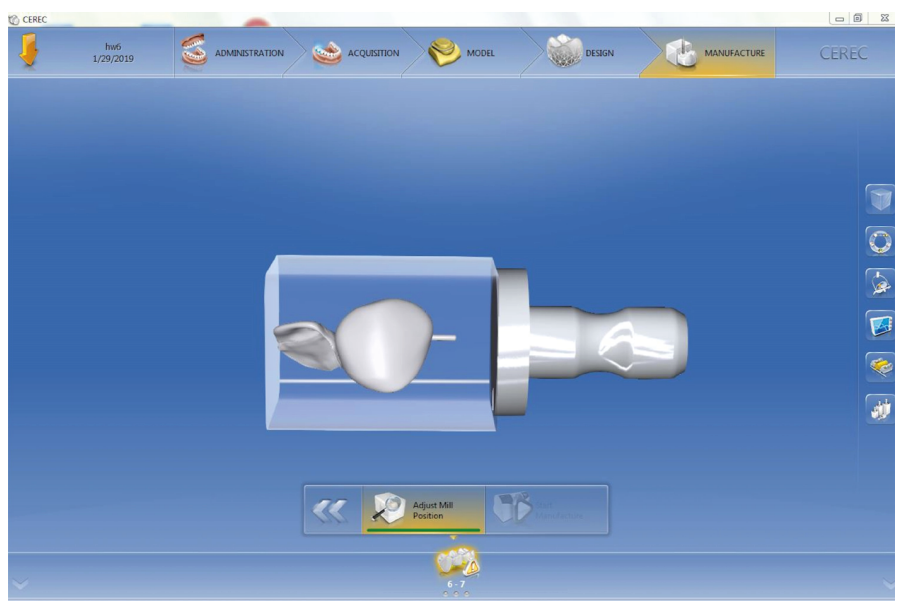

Figure 3. Digital design prior to milling.

denture, with the edentulous site as a pontic and the retainer tooth as a veneer. Following conventional scanning protocols, the upper and lower casts were scanned, having selected the edentulous area as the pontic and the lingual part of tooth \#7 as the veneer, as the software allows the clinician to select the position and dimension of the veneer on the lingual side. The software offers an ideal shape for both the veneer and pontic components of the restoration (Figure 2). The thickness of the restoration and occlusion were evaluated at this design stage, and the goal was to ensure at least $1 \mathrm{~mm}$ thickness for the veneer area and that the pontic unit was under occlusion. The final restoration had to fit within the CAD/CAM block, and due to its size, the sprue could only be placed on the interproximal side of the pontic (Figure 3 ). The final
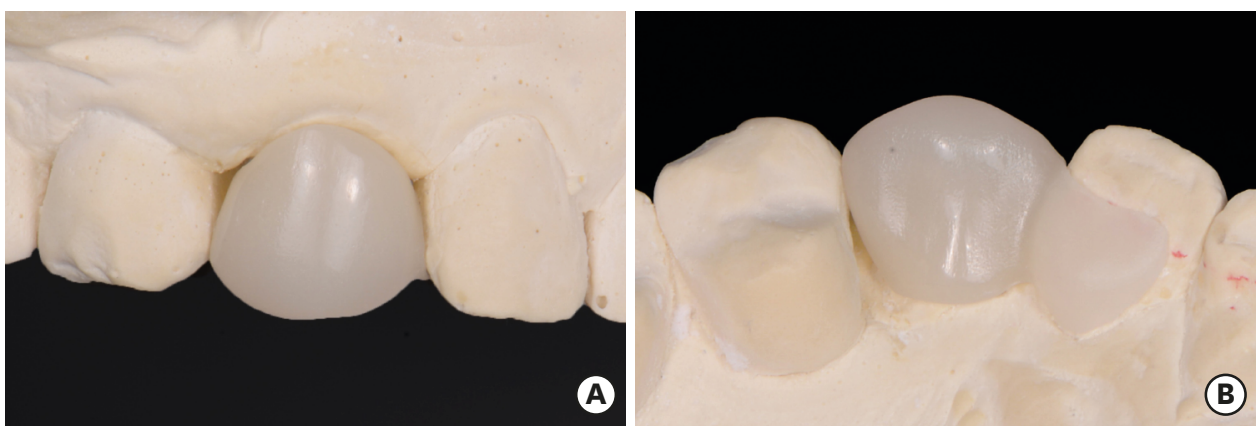

Figure 4. Final restoration try-in on the cast model. (A) Facial view of final restoration in cast. (B) Occlusal view of final restoration in cast 


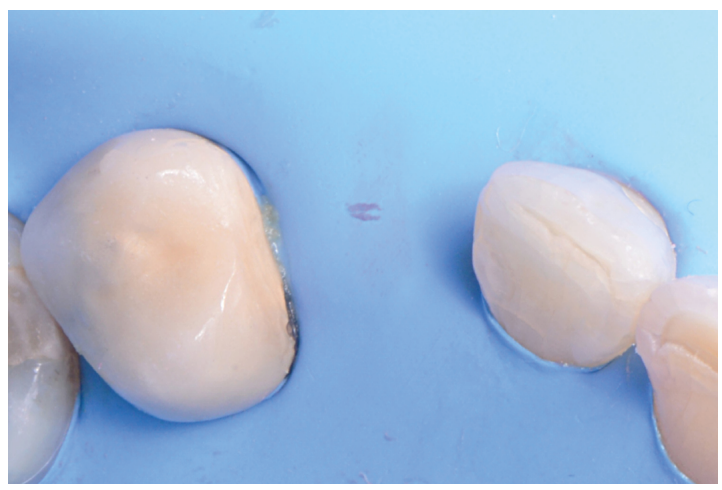

Figure 5. Occlusal view of isolation prior bonding.

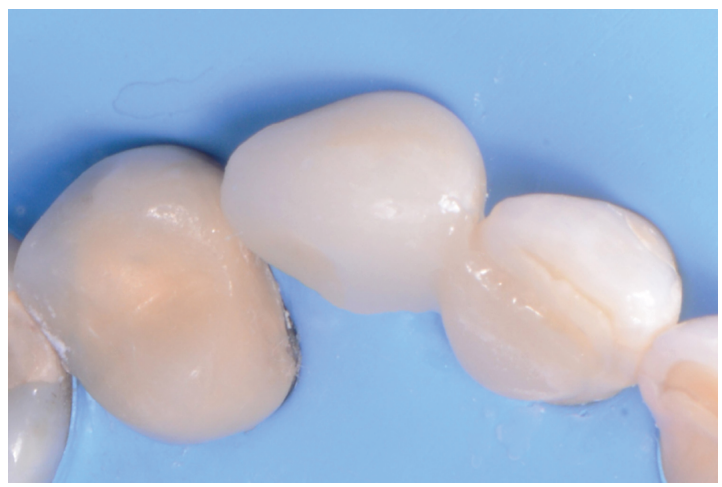

Figure 6. Occlusal view of bonded final restoration.

restoration was milled using lithium disilicate (e.max CAD, Ivoclar Vivadent) and placed in the master casts in order to evaluate occlusion and interproximal contacts (Figure 4).

The patient was referred to the Periodontic Department for the extraction of non-restorable tooth \#6 immediately after the impression and came back to the Restorative Department for bonding of the prepared single retainer cantilevered RBFDP to abutment tooth \#7 after the extraction. A rubber dam (Dental Dam, Nic Tone, Bucharest, Romania) was placed from tooth \#3 to tooth \#13 and retained with clamps (Clamp \#00, Hu-Friedy, Chicago, IL, USA) in order to achieve good isolation (Figure 5). The restoration was tried in prior to the bonding procedures. The adherend surface of the restoration was etched with $4.5 \%$ hydrofluoric acid (Ceramic Etching Gel, Ivoclar Vivadent) for 60 seconds, followed by rinsing and drying, then a pretreatment agent (Monobond Plus, Ivoclar Vivadent) was applied for 20 seconds. Surface treatment of the lingual side of tooth \#7 was completed prior to the bonding of the restoration. The enamel surface was etched with $37 \%$ phosphoric acid (Total Etch, Ivoclar Vivadent) for 15 seconds and gently air-dried, followed by the application of a primer (Multilink Primer, Ivoclar Vivadent) and gentle removal of the excess with air. The white shade of resin cement (Multilink Automix, Ivoclar Vivadent) was applied, and then the single retainer cantilevered RBFDP was bonded, followed by removal of the excess of the cement. The definitive restoration was light-cured on the lingual retainer surface for 60 seconds. Floss was used for removal of the excess cement in the interproximal area, followed by an additional light cure (60 seconds) on the mesial surface of the lingual retainer (Figure 6). The rubber dam was carefully removed and all remaining excess cement was removed. The patient was pleased with the contours, shape, and shade of the long-term chair-side CAD/CAM 


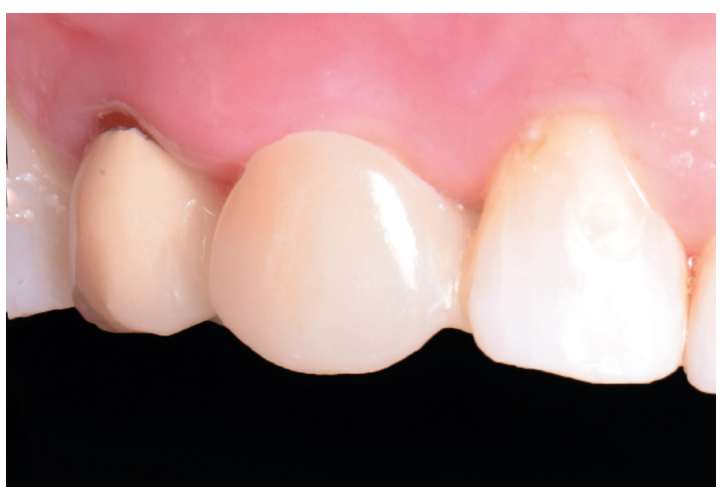

Figure 7. Facial view of the single-retainer resin-bonded fixed dental prosthesis 2 years after cementation.

RBFDP. The patient was recalled every 6 months, and no clinical problems or complications were found before or at the 2-year follow-up examination (Figure 7). Satisfactory functional and esthetic outcomes were achieved, and thus the patient decided not to proceed with implant therapy, rather retaining this restoration.

\section{DISCUSSION}

The main goal of this treatment was to provide a fixed restorative solution for the edentulous site \#6 that could meet the patient's esthetic demands and preserve the properly established mesiodistal space during tissue healing after root extraction and in preparation for future implant treatment without damage to proximal teeth. A secondary goal was to complete the treatment in a single day using a chair-side CAD/CAM system. The restoration was very conservative because the occlusion was evaluated intra- and extra-orally and it was possible to design, fabricate and deliver the restoration without tooth preparation. The maximum intercuspation position allowed enough lingual space for the single retainer on tooth \#7. Tooth \#5 had a porcelain fused to metal restoration that made the option of using it as a retainer challenging, and thus tooth $\# 7$ was used as the retainer. In addition, complete isolation with rubber dam provided a space between the prosthesis and the extracted site, which was bleeding. Because in this situation, the tooth was extracted, and the restoration was quickly fabricated and bonded without contamination of the bonding site.

Chair-side CAD/CAM RBDDPs can be scanned and designed directly intra-orally or on the master cast models, as in this case. Scanning and designing the restoration directly intra-orally can avoid any distortions due to cast fabrication, such as stone expansion, contamination made by impression materials and inaccuracies in the conventional impression. Extra-orally assessment of the lingual space for the single retainer and the adaptation of the prepared RBFDP to the abutment tooth could be conducted using the master cast models. In addition, the restoration was designed based on scanned data from the master cast models. In this case, to ensure that the preparations were all accomplished correctly, the restoration was fabricated from a laboratory scan of a model, but we anticipate future case reports in which the restoration is fabricated based on an intra-oral scan.

Conservative tooth preparation on the lingual side may help to seat the RBFDPs in the desired location properly. But since this type of restoration was intended for use as a provisional restoration during the healing of the tooth extraction site for future implant 
placement, we decided not to perform any tooth preparation to fix the restoration in this case. However, in this clinical case, the two-year follow-up demonstrated excellent stability of the RBFPD, and it was satisfactory for the patient.

A recent study using universal adhesive for bonding to unground enamel showed that there was no difference in bond strength to phosphoric acid etched unground and ground enamel [11]. In addition, hydrofluoric acid etching to lithium disilicate may improve resin cement bonding due to the creation of a microscale irregular structure on the surface [12]. Further, the rubber dam was able to isolate the lingual side of the retainer tooth in order to prevent any contamination from the extraction site, and thus the bonding properties both of the interfaces between enamel and adhesive, and resin cement and lithium disilicate were maximized. Because of these factors, a secure adhesion was obtained with a single retainer cantilever RBFDP fabricated by a chair-side CAD/CAM system resulting in a positive longterm prognosis. However, there is a lack of reported clinical trials comparing chair-side CAD/ CAM manufactured one- and two-retainer RBFDPs, and further investigations are needed.

This chair-side RBFDP was fabricated out of a lithium disilicate block. The authors initially planned to use a resin composite blocks, but the software does not allow this because the software regards this restoration as two units. Currently, resin composite blocks are not permitted for the fabrication of two or more splinted units. If a future software update made it possible to treat a similar case using a resin composite block, costs would be reduced, and it would be possible to complete the entire procedure, from the extraction of the tooth to the placement of the temporary bridge, at chair-side and on a single day.

\section{CONCLUSIONS}

The use of a chair-side CAD/CAM system for fabrication of a single-retainer RBDFPs allowed completion of the procedure in one day, and a single clinic visit, resulting in a functional and esthetic result that was still satisfactory after 2 years.

\section{REFERENCES}

1. Raigrodski AJ. Contemporary materials and technologies for all-ceramic fixed partial dentures: a review of the literature. J Prosthet Dent 2004;92:557-562. PUBMED | CROSSREF

2. Jurado CA, Tsujimoto A, Guzman LG, Fischer NG, Markham MD, Barkmeier WW, Latta MA. Implant therapy with ultratranslucent monolithic zirconia restorations in the esthetic zone: a case report. Gen Dent 2020;68:46-49. PUBMED

3. Bachhav VC, Aras MA. Zirconia-based fixed partial dentures: a clinical review. Quintessence Int 2011;42:173-182. PUBMED

4. Rochette AL. Attachment of a splint to enamel of lower anterior teeth. J Prosthet Dent 1973;30:418-423. PUBMED | CROSSREF

5. Howe DF, Denehy GE. Anterior fixed partial dentures utilizing the acid-etch technique and a cast metal framework. J Prosthet Dent 1977;37:28-31. PUBMED | CROSSREF

6. Livaditis GJ. Cast metal resin-bonded retainers for posterior teeth. J Am Dent Assoc 1980;101:926-929. PUBMED | CROSSREF 
7. Livaditis GJ, Thompson VP. Etched castings: an improved retentive mechanism for resin-bonded retainers. J Prosthet Dent 1982;47:52-58. PUBMED | CROSSREF

8. Botelho M. Resin-bonded prostheses: the current state of development. Quintessence Int 1999;30:525-534. PUBMED

9. Mourshed B, Samran A, Alfagih A, Samran A, Abdulrab S, Kern M. Anterior cantilever resin-bonded fixed dental prostheses: a review of the literature. J Prosthodont 2018;27:266-275. PUBMED | CROSSREF

10. Chen J, Cai H, Ren X, Suo L, Pei X, Wan Q. A systematic review of the survival and complication rates of all-ceramic resin-bonded fixed dental prostheses. J Prosthodont 2018;27:535-543. PUBMED | CROSSREF

11. Takeda M, Takamizawa T, Imai A, Suzuki T, Tsujimoto A, Barkmeier WW, Latta MA, Miyazaki M. Immediate enamel bond strength of universal adhesives to unground and ground surfaces in different etching modes. Eur J Oral Sci 2019;127:351-360. PUBMED | CROSSREF

12. Yoshida F, Tsujimoto A, Ishii R, Nojiri K, Takamizawa T, Miyazaki M, Latta MA. Influence of surface treatment of contaminated lithium disilicate and leucite glass ceramics on surface free energy and bond strength of universal adhesives. Dent Mater J 2015;34:855-862.

PUBMED | CROSSREF 